IV. Erfahrungen im Gespräch 


\section{Herausforderungen an eine Universität}

Hellmut Samonigg, befragt von Wolfgang Kröll

Kröll: Sehr geehrter Herr Rektor! Vielen Dank, dass Du Dir trotz der Belastung durch den Beruf und der Herausforderung durch die Corona-Pandemie Zeit genommen und dem Interview zugestimmt hast. Wie hast Du bzw. wie hat Deine Familie die Zeit der Pandemie, die Zeit der Lockdowns, die Zeit der sozialen Isolation, die Zeit der zahlreichen Einschränkungen erlebt? Ich hoffe, dass Du und Deine Angehörigen auch gesund durch diese Krisenzeit gekommen sind. Bist $D u$ bereits geimpft und falls ja: Wie hast Du die Impfung vertragen? Sind irgendwelche Nebenwirkungen, die wiederum per se zu persönlichen Einschränkungen geführt haben, aufgetreten?

Samonigg: Da ich Mitglied der Klinikumsleitung bin, wurde ich auch bereits im ersten Quartal 2021 geimpft; ich wurde mit einem Impfstoff von Pfizer geimpft und habe beide Impfungen auch sehr gut vertragen; ich bin sogar mehr oder weniger unmittelbar nach der Impfung zum Schifahren gefahren; dort litt ich dann am darauffolgenden Tag an einem Schnupfen; aber nicht als Nebenwirkung des Impfstoffes, sondern weil die Liftanlage ausgefallen ist und ich etwa 45 Minuten bei minus 15 Grad Celsius am Lift festgesessen bin. Ich bin überzeugt, dass dieser Schnupfen nichts mit der Impfung zu tun hatte. Nach der zweiten Impfung war ich dann nicht mehr so forsch und es sind danach überhaupt keine Symptome aufgetreten.

Ich bin über diese gesamte Phase der Pandemie sehr gut hinweggekommen, aber es war für mich persönlich auch wichtig, dass ich jeden Tag in mein Büro gehen konnte und meine Arbeit, die ich eigentlich, zumindest zum größten Teil, auch im Home-Office hätte erledigen können, an meinem Arbeitsplatz an der Medizinischen Universität Graz (MUG) erledigen konnte. Das war für mich einfach psychologisch wichtig, dass ich hier an der MUG war.

Durch die Verordnungen der Bundesregierung wurden natürlich auch die Sozialkontakte eingeschränkt, aber das war für mich nicht von großer Bedeutung, da ich nicht jeden Abend in irgendein Restaurant gehe, sondern auch meine Zeit gerne im Kreise der Familie zu Hause verbringe. Aber irgendwann ist dann schon der Augenblick gekommen, wo ich auch 
daran gedacht habe, dass es jetzt an der Zeit wäre in ein Restaurant zu gehen, um gemütlich mit Freunden und Bekannten zu speisen.

In meinem familiären Umfeld hat es keine Infektionen mit SARS-CoV2 gegeben; auch nicht bei uns im Rektorat, sehr wohl aber in den einzelnen Kliniken, aber dort waren diese Infektionen bei den Mitarbeitern, soweit ich darüber informiert bin, gut beherrschbar. Zusammenfassend kann man festhalten, dass die MUG und das LKH-Univ.-Klinikum sehr gut durch die Corona-Pandemie gekommen sind.

Kröll: Die Pandemie ist plötzlich über Europa hereingebrochen und hat uns alle mehr oder weniger überrascht und möglicherweise auch auf dem falschen Fuß getroffen. Die MUG war von einem auf den anderen Tag gefordert, einerseits die Ausbildung der Studierenden - gemeint sind Vorlesungen und Prüfungen weiterhin und trotzdem zu gewährleisten, andererseits aber die entsprechenden Bestimmungen des Gesetzgebers zu beachten. Wie ist es Dir und Deinem Team dabei gegangen, dies alles in kürzester Zeit zu bewerkstelligen? War die MUG darauf vorbereitet?

Samonigg: Dadurch, dass die MUG bereits seit vielen Jahren den virtuellen Campus (E-Learning) eingeführt hat, haben die Lehrenden, bis auf etwa 15 Prozent, bereits gute Erfahrung damit sammeln können. Das war natürlich sehr hilfreich, weil wir bereits in den ersten beiden Wochen nach Verhängung des Lockdowns auf dieses System umstellen und dieses auch erweitern konnten, sodass eben die meisten lehrenden Kollegen keinen Stress mit dieser Notwendigkeit, die Lerninhalte über diese Plattform weiterzugeben, hatten. Insgesamt wurde dieser Bereich des E-Learnings um das sechsfache gesteigert. Aus meiner derzeitigen Sicht wird ungefähr ein Anteil von 35 Prozent an E-Learning auch nach der Pandemie fester Bestandteil der Vermittlung von Lerninhalten an unserer Universität bleiben. Dass ein Teil, nämlich die bereits angesprochenen 15 Prozent, keine Erfahrung mit E-Learning hatten, wurde von den Studierenden natürlich sehr schnell wahrgenommen und sie haben sich darüber auch erheblich echauffiert.

Die Studierenden waren verpflichtet bei Präsenzveranstaltungen - und das hat während der Corona-Pandemie ca. 10-15 Prozent aller Lehrveranstaltungen ausgemacht, z. B. erforderlich durch die diversen klinischen Praktika - negative Antigentests vorzuweisen; das hat aber zu überhaupt keiner ablehnenden Haltung seitens der Studierenden geführt. Bei den Präsenzveranstaltungen des Univ.-Instituts für Anatomie waren sogar PCR-Tests verpflichtend; die Durchführung dieser Untersuchung hat die MUG selbst organisiert. Und auch diese Vorgaben sind von den Studieren- 
den ohne negative Kommentare eingehalten worden und haben sich auch problemlos organisieren lassen.

An der MUG sind nicht nur ein hoher Prozentsatz der Mitarbeiter geimpft, sondern auch ein großer Teil unserer Studierenden; ich schätze, dass die Durchimpfungsrate bei Mitarbeiterinnen und Studierenden etwa bei 75 Prozent liegt. Ich tendiere aber dazu sowohl die nicht-geimpften Mitarbeiter als auch die nicht-geimpften Studierenden noch einmal zu motivieren sich auch impfen zu lassen. Und bei Neueinstellungen verlange ich, dass der neue Mitarbeiter bereits geimpft ist.

Ich bin grundsätzlich ein Befürworter der Impfpflicht, man hat es ja auch bei anderen Infektionserkrankungen und Pandemien, wie z. B. bei den Pocken, so gemacht und hatte es dann erreicht die Erkrankung in 15 Jahren vollständig bekämpfen zu können. Ob das bei SARS-CoV2 auch so sein wird, weiß ich natürlich nicht. Und selbstverständlich können Nebenwirkungen auftreten, aber im Vergleich zur Bedrohung der Welt durch das Corona-Virus sind diese Nebenwirkungen aus meiner Sicht zu akzeptieren.

Kröll: Betroffen von diesen Einschränkungen bzw. Änderungen des universitären Betriebes und des Studiums waren aber nicht nur die Vortragenden, betroffen davon waren in zumindest gleichem Ausmaß auch die Studierenden. Wie war die Akzeptanz dieser Gruppe hinsichtlich des Studiums mit veränderten und teilweise auch eingeschränkten Möglichkeiten? Hat sich auch hier eine Entwicklung hin zur größeren Akzeptanz dieser Notwendigkeiten gezeigt? Wie war die Zufriedenheit der Studierenden mit diesen Bedingungen und wie war die Zufriedenheit mit dem Lehr- und Prüfungsangebot aus der Distanz?

Samonigg: Grundsätzlich sind sich auch die Studierenden darüber einig, dass zumindest ein Teil der Lehre auch in Zukunft als E-Learning abgehalten werden sollte. Aber natürlich wollen die Studierenden auch wieder den Präsenzunterricht. Und der muss auch wieder kommen, der ist bei einer medizinischen Universität nicht wegzudenken.

Bzgl. der Organisation der Lehre bilden die Universitäten seit Beginn der Corona-Pandemie eine Ausnahme; die Universitäten waren im Prinzip von den gesamten Regelungen, die Unterrichtsgestaltung betreffend, immer ausgenommen. Das hatte natürlich auch den Vorteil, dass die Universitäten ihr Vorgehen selbstbestimmt und mit Hausverstand regeln konnten. Diese Möglichkeit wurde auch von den meisten Rektoren der österreichischen Hochschulen und Universitäten sehr geschätzt; aber es hat natürlich auch Rektoren gegeben, die sich durch diese Ausnahmeregelung zu wenig geschützt gefühlt haben. Ich persönlich war über diese 
Möglichkeit sehr froh, weil ich dadurch, den Bedürfnissen entsprechend, selbständig entscheiden konnte.

Und die Studierenden wussten dieses Vorgehen an unserer MUG auch zu schätzen; es sind zu keinem Zeitpunkt irgendwelche Beschwerden aufgetreten.

Kröll: Die Universität lebt ja auch aus der Teilnahme der wissenschaftlichen Mitarbeiter an Tagungen und Kongressen und der Präsentation von Ergebnissen der eigenen wissenschaftlichen Forschung. Wie haben es die Kolleginnen hingenommen, dass plötzlich Dienstreisen nicht mehr möglich waren, dass, falls überhaupt angeboten, Tagungen und Kongresse digital abgehalten worden sind und Präsentationen mehr oder weniger obne Auditorium stattgefunden haben? Ist es jetzt den Mitarbeitern der MUG wieder erlaubt an Kongressen, sofern diese bereits in Präsenz angeboten werden, teilzunehmen? Bzw. ab wann denkst Du wird dies an der MUG möglich sein?

Samonigg: Die Corona-Pandemie hat auch organisatorisch einiges innerhalb des Betriebsablaufes, auch meines eigenen Betriebsablaufes, verändert. Als Rektor war ich vor Ausbruch der Corona-Pandemie beruflich sehr viel unterwegs, in Österreich, aber auch im Ausland. Diese Dienstreisen sind nun von heute auf morgen weggefallen und es hat sich alles auf die digitale Kommunikation verlagert. Das hatte den Vorteil, dass ich nicht mehr so häufig unterwegs war, aber der Nachteil dieser digitalen Kommunikation war, dass sich eine Video-Konferenz an die nächste gereiht hatte und das war aus meiner Sicht wesentlich anstrengender als die diversen Dienstreisen. Denn trotz der teilweise langen Autofahrten gab es einen gewissen Erholungseffekt, der war nun plötzlich weg. Aber man muss dennoch positiv bemerken, dass die Effektivität dieser digitalen Kommunikation sehr zielführend war im Hinblick auf die Durchführung und Bearbeitung von Prozessen und man auf diese Art und Weise auch Prozesse viel schneller zum Abschluss bringen konnte.

Wie zu erwarten hat es natürlich das Bestreben einiger weniger Mitarbeiterinnen gegeben auch während der Lockdown-Phasen zu Seminaren und Kongressen, auch ins Ausland, zu reisen. Teilweise waren diese Wünsche irrational: So gab es Fälle, wo jemand zu einer wichtigen Projektbesprechung mit zwei anderen Teilnehmern aus europäischen Ländern unbedingt in die Schweiz reisen wollte; erst als ich nachweisen konnte, dass keiner der anderen beiden Projektmitarbeiter an dieser Veranstaltung teilnehmen wird und diese Veranstaltung bereits seit längerer Zeit abgesagt ist, war man dann vernünftig genug auf die Teilnahme zu verzichten. 
Aber grundsätzlich waren die Mitarbeiterinnen so vernünftig, dass sie die Notwendigkeit an Seminaren, Symposien und Kongressen ausschließlich digital teilzunehmen eingesehen haben und sich auch an diese Anordnungen gehalten haben.

Ich selbst wundere mich ja, wie es den Veranstaltern von großen Kongressen mit internationaler Beteiligung gelungen ist, diese über die Bühne zu bringen; eine großartige Leistung, wenn man bedenkt, welche Schwierigkeiten bereits aufgetreten sind, wenn man diese Digitalisierung nur im kleinen Kreis durchführen wollte und musste.

Aber dennoch wird auch nicht alles digital möglich sein: Bewerbungsgespräche digital sind aus meiner Sicht insofern nicht möglich, weil man keinen richtigen Eindruck vom Bewerber erhält, wenn sich dieser nur über einen Bildschirm präsentiert. Das hat sich auch bei uns an der MUG gezeigt.

Kröll: Die MUG lebt aber nicht nur von der Präsentation der eigenen wissenschaftlichen Ergebnisse, sondern die MUG lebt, wie jede andere Universität auch, von den Forschungsleistungen ihrer Mitarbeiter. Nun wurden zwar in der Ethikkommission zahlreiche Studien auch in der Zeit der Pandemie und der Lockdowns eingereicht, aber wie stand es mit der Durchführung dieser Studien? War es den Kolleginnen möglich trotz all der Einschränkungen diese geplanten klinischen Prüfungen durchzuführen? War es, und vor allem unter welchen Auflagen, möglich insbesondere ambulante Probanden in die Klinik einzuschleusen, um die geplanten Untersuchungen im Rabmen der Prüfungen vorzunehmen?

Samonigg: Interessant zu bemerken ist, dass sich bei den Zielvereinbarungsgesprächen gezeigt hat, dass sich die Publikationstätigkeit der wissenschaftlichen Mitarbeiter durchgehend erhöht hat. Ich führe das darauf zurück, dass die Mitarbeiter einfach mehr Zeit zur Verfügung gehabt haben für die wissenschaftliche Tätigkeit, denn für viele wissenschaftliche Mitarbeiter ist die klinische Belastung weitgehend weggefallen bzw. hat sich auf nur wenige Gruppen an Mitarbeitern konzentriert: $\mathrm{Zu}$ nennen ist die Klinische Abteilung für Angiologie an der Univ.-Klinik für Innere Medizin, die eine eigene Station für COVID-19-Erkrankte zu betreuen hatte, zu nennen natürlich auch die Mitarbeiter jener klinischen Abteilungen, die COVID-19-Patienten auf Intensivstationen zu betreuen hatten, wie eben die Kolleginnen der Univ.-Klinik für Innere Medizin und der Univ.-Klinik für Anästhesiologie und Intensivmedizin; auch die Klinische Abteilung für Onkologie und Palliativmedizin war sehr involviert in die Behandlung von COVID-19-Patienten; einerseits waren teilweise die Mitarbeiter infiziert, andererseits hatten sie auch viele Patienten zu versorgen, 
die im Grunde alle als Risikopatienten zu qualifizieren waren und viele von diesen Patienten sind schließlich auch verstorben. Hingegen wurde in vielen anderen Univ.-Kliniken bzw. klinischen Abteilungen das Behandlungsangebot sehr drastisch reduziert und daher war es den wissenschaftlichen Mitarbeitern natürlich auch möglich vermehrt der wissenschaftlichen Tätigkeit nachzugehen.

Kröll: Die Pandemie scheint langsam zu Ende zu gehen. Nun wurden an der MUG zablreiche Neuerungen, bedingt durch die verordneten Einschränkungen des Lehr- und Forschungsbetriebes, installiert, die sich zumindest während dieses Zeitraumes sebr bewährt haben. In den Printmedien vom 3. Juni 2021 fordern die Studierenden, dass diese getroffenen Maßnahmen beibehalten werden. Wie siehst Du das und welche gesetzten Veränderungen werden an der MUG weiterhin für die Studierenden angeboten? Wie wirken sich diese Veränderungen im Lehr- und Forschungsbetrieb auf den Personalbedarf der wissenschaftlichen Mitarbeiter aus? Findest Du mit dem derzeitigen Stand an ärztlichen Vollzeitäquivalenten das Auslangen? Benötigst Du mehr wissenschaftliches Personal oder musst Du, bei Weiterbestand der eingeführten Neuerungen, sogar Mitarbeiter entlassen?

Samonigg: Es wird einiges von dem, was die Corona-Pandemie gebracht hat, auch nach Ende der Pandemie weiterhin Bestand haben: Wenn auch nicht in vollem Umfang, so wird Distance-Learning weiterhin auf der MUG einen breiteren Raum einnehmen als bisher. Vergleichbares gilt auch für das Home-Office; aber auch da muss man Grenzen ziehen, denn es sind bereits die ersten Anfragen eingetroffen, die eine vertragliche Festlegung für zwei Tage Home-Office pro Woche anstreben; und da sollten dann noch Wunschtage, nämlich Montag und Freitag, berücksichtigt werden. Da muss ein Riegel vorgeschoben werden; ich kann mir einen Tag Home-Office für die wissenschaftlichen Mitarbeiterinnen vorstellen und der wird nach Rücksprache mit dem jeweiligen Abteilungsleiter vergeben werden.

Für die Belange der MUG haben wir ausreichend Mitarbeiter, auch trotz oder vielleicht gerade wegen der Neuerungen, die sich durch die Corona-Pandemie ergeben haben. Und da ja auch der medizinische Betrieb wieder voll im Gange ist besteht auch keine Notwendigkeit irgendeinen Mitarbeiter zu entlassen.

Kröll: Sehr geehrter Herr Rektor! Vielen Dank für Deine Bereitschaft diese ausfübrlichen Fragen zu beantworten. 